\title{
Hybrid Algorithm for Medical Image Sequences using Super-Spatial Structure Prediction with LZ8
}

\author{
M.Ferni Ukrit \\ Research Scholar \\ Department of CSE \\ Sathyabama University \\ Chennai
}

\author{
G.R.Suresh \\ Professor \\ Department of ECE \\ Easwari Engineering College \\ Chennai
}

\begin{abstract}
The necessity in medical image compression continuously grows during the last decade. In advanced medical life large number of medical images is processed in hospitals and medical centers around the world. These images are in the form of sequences which are much correlated and are of great importance. Hence lossless image compression is needed to reproduce the original quality of the image without any loss of information. To exploit the correlation a new algorithm is proposed in this paper. The proposed compression method combines Super-Spatial Structure Prediction with motion estimation and motion compensation to achieve higher compression ratio. This is applied with a simple blockmatching process Binary Tree Search. Results are compared in terms of Compression Ratio and Peak Signal-to-Noise Ratio. The proposed methodology provides better CR and PSNR than the other state-of-the-art algorithm.
\end{abstract}

\section{Keywords}

Medical Image Sequences, Super-Spatial Structure Prediction, Lossless Compression, Motion Estimation and Motion Compensation, Inter-frame Coding, CALIC, LZ8

\section{INTRODUCTION}

Hospitals and various medical organizations produce huge volume of digital medical image sequences which includes Computed Tomography (CT), Magnetic Resonance Image (MRI), Ultrasound and Capsule Endoscope (CE) images. These medical image sequences require considerable storage space [1].The solution to this problem could be the application of compression. Medical image compression is very important in the present world for efficient archiving and transmission of images. Image compression can be classified as lossy and lossless. In lossy compression scheme there is loss of information and the original image is not recovered exactly. Lossy scheme seems to be irreversible. But lossless scheme is reversible and this represents an image signed with the smallest possible number of bits without loss of any information thereby speeding up transmission and minimizing storage requirement. Lossless reproduces the original image without any quality loss [2].Medical imaging does not require lossy compression due to the following reason. The first reason is the incorrect diagnosis due to the loss of useful information. The second reason is the operations like image enhancement may emphasize the degradations caused by lossy compression. Hence efficient lossless compression methods are required for medical images [3].Lossless compression includes Discrete Cosine Transform, Wavelet Compression, Fractal Compression, Vector Quantization and Linear Predictive Coding. Lossless consist of two distinct and independent components called modeling and coding. The modeling generates a statistical model for the input data. The coding maps the input data to bit strings [4].
Several Lossless image compression algorithms were evaluated for compressing medical images. There are several lossless image compression algorithms like Lossless JPEG,JPEG 2000,PNG,CALIC and JPEG-LS.JPEG-LS has excellent coding and best possible compression efficiency[1].But the Super-Spatial Structure Prediction algorithm proposed in [5] has outperformed the JPEG-LS algorithm. This algorithm divides the image into two regions, structure regions (SRs) and non-structure regions (NSRs). The structure regions are encoded with Super-Spatial Structure Prediction technique and non-structure regions are encoded using JPEG-LS. The idea of Super-Spatial Structure Prediction is taken from video coding. There are many structures in a single image. These include edges, pattern and textures. This has relatively high computational efficiency. No codebook is required in this compression scheme because the structure components are searched within the encoded image regions [6]. JPEG-LS has excellent coding and best possible compression efficiency [1].This is a simple and baseline algorithm which consists of two independent and distinct stages called modeling and encoding. This is developed with the aim of providing a low complexity lossless and nearlossless image compression. This is based on LOCO-I (Low Complexity Lossless Compression for Images) algorithm [7] using adaptive prediction, context, modeling and Golomb coding. It supports near lossless compression by allowing a fixed maximum sample error. A continuous image is generally compressed best in JPEG-LS [8].

Most of the lossless image compression algorithms take only a single image independently without utilizing the correlation among the sequence of frames of MRI or CE images. Since there is too much correlation among the medical image sequences, we can achieve a higher compression ratio using inter-frame coding. The idea of compressing sequence of images was first adopted in [9] for lossless image compression and was used in [10], [11], [12] for lossless video compression. The Compression Ratio (CR) was significantly low (i.e.) 2.5 which was not satisfactory. Hence in [1] they have combined JPEG-LS with inter-frame coding to find the correlation among image sequences and the obtained ratio was 4.8.Super-Spatial Structure Prediction algorithm proposed in [13] has outperformed JPEG-LS. However this ratio can be enhanced using Super-Spatial Structure Prediction technique and LZ8.Super-Spatial Structure Prediction is applied with a simple block matching algorithm Binary Tree Search (BTS) [13]. LZ8 is used to further compress the output code of SSP [14].

In this paper, we propose a hybrid algorithm for medical image sequences. The proposed algorithm combines SuperSpatial Structure Prediction technique with MEMC and a new innovative scheme LZ8 to achieve a high compression ratio. The Compression Ratio (CR) can be calculated by the equation (1) and PSNR by equation (2) 
PSNR $=20 * \log 10(255 \mid$ sqrt $($ MSE $))$

This paper is organized as follows:

Section 2 explains the methodology used which includes Overview, Super-Spatial Structure Prediction, Motion Estimation and Motion Compensation, Motion Vector, Block Matching Algorithm and LZ8. Section 3 discusses the results obtained for the proposed methodology.

\section{METHODOLOGY USED}

\subsection{Overview}

The objective of the proposed method is to enhance the compression efficiency using Super-Spatial Structure Prediction (SSP) technique combined with Motion Estimation and Motion Compensation (MEMC). The compression ratio is further enhanced by LZ8 algorithm. Fig. 1 illustrates the complete encoding technique of the proposed method.

Given an image sequence, input the first image to be compressed. The images are classified to Structure Regions (SRs) and Non-Structure Regions (NSRs). The first image will be compressed by Super-Spatial Structure Prediction algorithm since there is no reference frame. After the first image is compressed the second frame will be the current frame and the first frame becomes the reference for the second frame. Inter-frame coding includes MEMC process to remove temporal redundancy. Inter coded frame will be divided to blocks known as macro blocks. The encoder will try to find a similar block as the previously encoded frame. This process is done by a block matching algorithm called Binary Tree Search (BTS). If the encoder succeeds on its search the block is directly encoded by a vector known as Motion Vector. After MEMC is done the difference of images is processed for compression. The difference is also compressed using SuperSpatial Structure Prediction compression. The Motion Vectors (MV) derived from motion estimation will also be compressed. The output code of SSP is compressed by replacing the repeated occurrences of data with references to a dictionary that is built based on the input data stream. Then the flag bits and the encoded bits for one image frame are concatenated into single bit stream. Once the compression of the second frame is done it becomes the reference frame for the third frame and this processing will be repeated for the next image until the end of image sequence.

\subsection{Super-Spatial Structure Prediction}

Super-Spatial Structure Prediction borrows its idea from motion prediction [15].In SSP an area is searched within the previously encoded image region to find the prediction of an image block. The reference block that results in the minimum block difference is selected as the optimal prediction. Sum of Absolute Difference (SAD) is used to measure the block difference. The size of the prediction unit is an important parameter. When the size is small the amount of prediction and coding overhead will become large. If larger prediction unit is used the overall prediction efficiency will decrease. In this paper, a good substitution between this two is proposed. The image is partitioned into blocks of $8 \times 8$ and classifies these blocks to structure and non-structure blocks. Structure blocks are encoded using SSP and non-structure blocks using JPEG-LS.This is based on LOCO-I (Low Complexity Lossless Compression for Images) algorithm using adaptive prediction, context, modeling and Golomb coding.

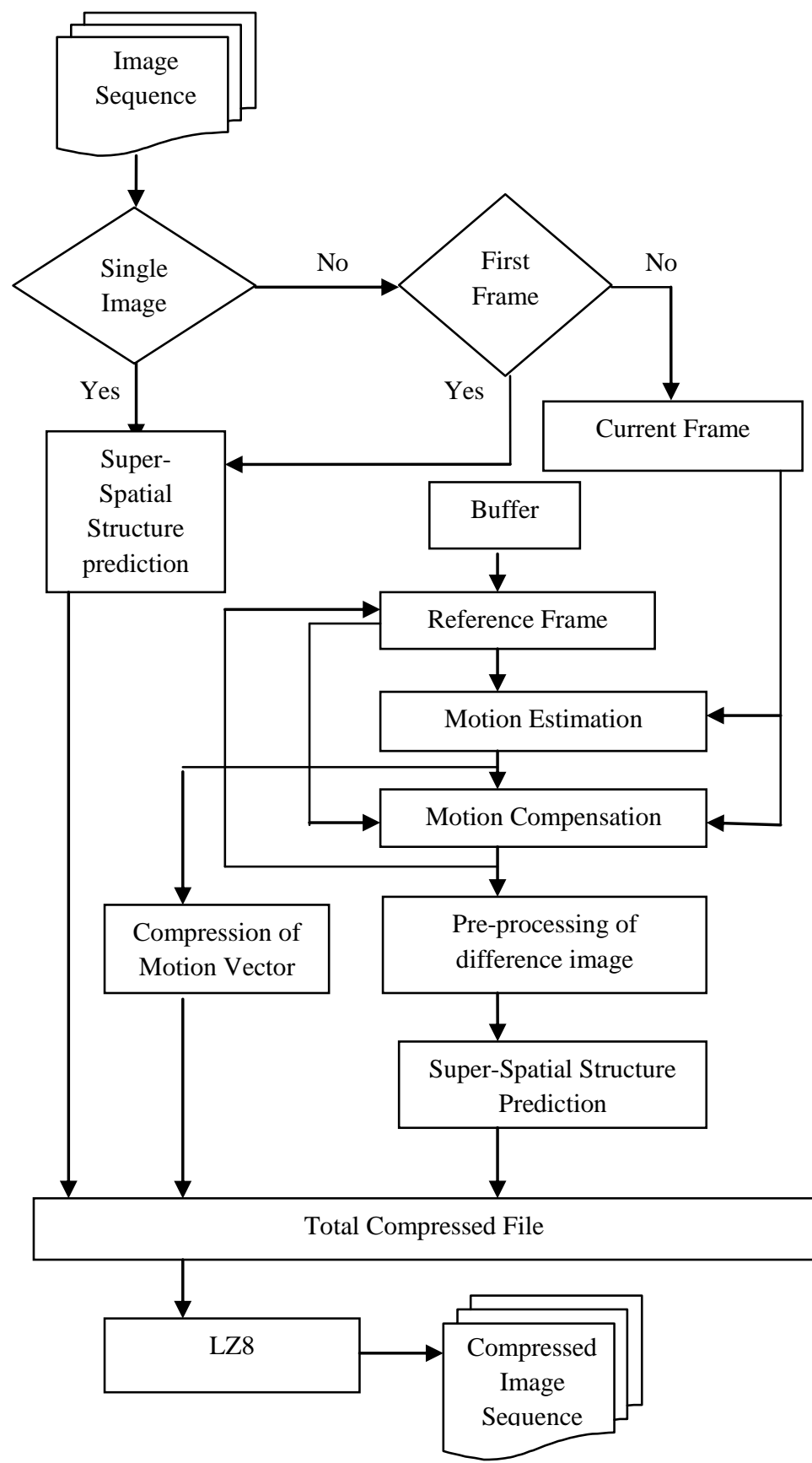

Fig. 1 Encoding Technique of the Proposed Method

It supports near lossless compression by allowing a fixed maximum sample error. The image is classified to SRs and NSRs and then SSP is applied to SRs since its prediction gain in the non structure smooth regions will be very limited. This will reduce the overall computational complexity [5].

\subsection{MEMC}

Motion estimation is the estimation of the displacement of image structures from one frame to another in a time sequence of $2 \mathrm{D}$ images. The displacement vector at location $r$ in frame at time $\mathrm{t}, \mathrm{d}$ describing the motion from frame at $\mathrm{t}$ to frame at $\mathrm{t}+\Delta \mathrm{t}$, as

$$
d(r)=\left[d_{x}(r) d_{y}(r)\right]^{T}
$$


where $\mathrm{r}=[\mathrm{x}, \mathrm{y}] \mathrm{T}$, the continuous spatial coordinates and superscript $\mathrm{T}$ denotes vector transposition. The dependency of the displacement vector on spatial coordinates for each location $\mathrm{r}$ at frame $\mathrm{t}$, a displacement vector can be defined as in Fig 2.

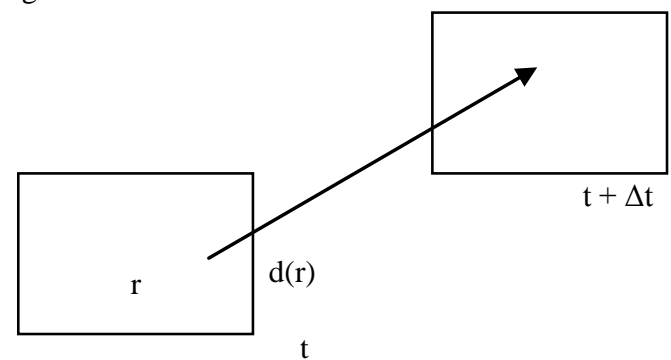

Fig. 2 Structure of motion estimation

The steps in MEMC is stated as

- Find displacement vector of a pixel or a set of pixels between frame

- Via displacement vector, predict counterpart in present frame

- Prediction error, positions, motion vectors are coded \& transmitted

The Fig.3.illustrates the block diagram of motion compensated coding.

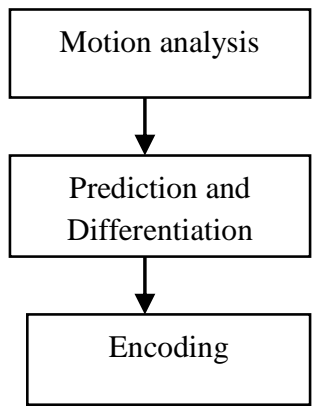

\section{Fig. 3 Block diagram of motion compensated coding}

Motion estimation can be very computationally intensive and so this compression performance may be at the expense of high computational complexity. The motion estimation creates a model by modifying one or more reference frames to match the current frame as closely as possible. The current frame is motion compensated by subtracting the model from the frame to produce a motion-compensated residual frame. This is coded and transmitted, along with the information required for the decoder to recreate the model (typically a set of motion vectors). At the same time, the encoded residual is decoded and added to the model to reconstruct a decoded copy of the current frame (which may not be identical to the original frame because of coding losses).This reconstructed frame is stored to be used as reference frame for further predictions. The inter-frame coding should include MEMC process to remove temporal redundancy. Difference coding or conditional replenishment is a very simple inter-frame compression process during which each frame of a sequence is compared with its predecessor and only pixels that have changes are updated. Only a fraction of pixel values are transmitted. An inter-coded frame will finitely be divided into blocks known as macro blocks. After that, instead of directly encoding the raw pixel values for each block, as it would be done for an intra-frame, the encoder will try to find a similar block to the one it is encoding on a previously encoded frame, referred to as reference frame. This process is done by a block matching algorithm. [14]. If the encoder succeeds on its search, the block could be directly encoded by a vector known as motion vector, which points to the position of the matching block at the reference frame.

\subsection{Motion Vector}

Motion estimation is using a reference frame in a video, dividing it in blocks and figuring out where the blocks have moved in the next frame using motion vectors pointing from the initial block location in the reference frame to the final block location in the next frame. For MV calculation we use Block matching algorithm as it is simple and effective. It uses Mean Square Error (MSE) for finding the best possible match for the reference frame block in the target frame. Motion vector is the key element in motion estimation process. It is used to represent a macro block in a picture based on the position of this macro block in another picture called the reference picture. In video editing, motion vectors are used to compress video by storing the changes to an image from one frame to next. When motion vector is applied to an image, we can synthesize the next image called motion compensation [9],[13]. This is used to compress video by storing the changes to an image from one frame to next frame. To improve the quality of the compressed medical image sequence, motion vector sharing is used. [12].

\subsection{Block Matching}

In block matching technique, each frame is divided into equalsize blocks, called source blocks.Each source block is associated with a search region in the reference frame.The objective of block-matching is to find a candidate block in the search region best matched to the source block. The relative distances between a source block and its candidate blocks are called motion vectors. The block-matching process during the function MEMC taken from [1] takes much time hence we need a simple searching method and we have taken Binary Tree Search (BTS) method [13] .In order to speed up the block matching process, the blocks having the same sum are compared and the others are left. Therefore the time taken is reduced. This process works as follows:

Step 1: Input the block.

Step 2: If it is the first block insert it into the first index of the block array

Step 3: Insert the block sum and its index in the root node of the binary tree.

Step 4: If it is not the first block then calculate its sum and search over the Binary tree.

Step 5: If the sum is found then find if there is any matching block, if found record the index of the block and no new block is inserted and no node is created in the Binary tree.

Step 6: If the sum is not found and match also not found then insert block into the array and a new node is created in the Binary tree.

Step 7: Repeat the process until the last block.

The Algorithm is discussed:

Require: Image(512x512)-> I, Refblock(4x4)->Rf,

Search area $(4 \times 4=16)->\mathrm{Sa}$

Sb->search block

For S1-> height

For S2--> width

$\mathrm{Sb}=\mathrm{I}(\mathrm{S} 1, \mathrm{~S} 2)$

$[$ Dist, idx, bestblock] $=\mathrm{SAD}(\mathrm{Rf}, \mathrm{Sb})$

$\mathrm{Fbb}=$ bestblock 
If bestblock already found Fbb

Keep previous $\mathrm{Fbb}$

End if

End for

End for

SAD Process returns->Dist, idx, bsetblock

Dist $=$ Sort $($ Dist $)$

Bestblock $=$ best block selection

The formula for SAD is given by the equation (4) and MSE is calculated by equation (5)

$$
\begin{aligned}
& S A D=\sum_{(i, j) \exists W}|I 1(i, j)-I 2(x+i, y+j)| \\
& M S E=\frac{1}{M * N} \sum_{i=0}^{M} \sum_{j=0}^{N}[I 1(m, n)-I 2(m, n)]^{22}
\end{aligned}
$$

Where $\mathrm{M}$ and $\mathrm{N}$ are the number of rows and columns in the input images. I1 is the original image and $\mathrm{I} 2$ is the decompressed image.

\subsection{LZ8}

LZ8 is an LZ78-based algorithm that uses a dictionary preinitialized with all possible characters (symbols), (or emulation of a pre-initialized dictionary). The main improvement of LZ8 is that when a match is not found, the current input stream character is assumed to be the first character of an existing string in the dictionary (since the dictionary is initialized with all possible characters), so only the last matching index is output (which may be the preinitialized dictionary index corresponding to the previous (or the initial) input character).

LZ8 algorithm achieves compression by replacing repeated occurrences of data with references to a dictionary that is built based on the input data stream. Each dictionary entry is of the form dictionary $[\ldots]=\{$ index, character $\}$, where index is the index to a previous dictionary entry, and character is appended to the string represented by dictionary [index]. For example, "abc" would be stored (in reverse order) as follows: dictionary $[\mathrm{k}]=\left\{\mathrm{j}, \mathrm{c}^{\prime}\right\}$, dictionary $[\mathrm{j}]=\left\{\mathrm{i}, \mathrm{b}^{\prime}\right\}$, dictionary $[\mathrm{i}]=$ $\{0$, 'a' $\}$, where an index of 0 implies the end of a string. The algorithm initializes last matching index $=0$ and next available index $=1$. For each character of the input stream, the dictionary is searched for a match: \{last matching index, character\}. If a match is found, then last matching index is set to the index of the matching entry, and nothing is output. If a match is not found, then a new dictionary entry is created: dictionary[next available index $]=$ [last matching index, character\}, and the algorithm outputs last matching index, followed by character, then resets last matching index $=0$ and increments next available index. Once the dictionary is full, no more entries are added. When the end of the input stream is reached, the algorithm outputs last matching index. Note that strings are stored in the dictionary in reverse order, which a LZ78 based decoder will have to deal with.[16].

The first frame is decompressed using LZ8 followed by Super-Spatial Structure Prediction decoder. After the reproduction of the first frame the difference of the rest of the frames are decompressed. The first frame becomes the reference frame for the next frame. After the reproduction of the second frame it becomes the reference frame for the next frame and the process continues until all the frames are decompressed.

\section{RESULTS AND DISCUSSION}

The proposed methodology has been simulated in Microsoft Visual Studio.Net 2005. The performance of the proposed methodology is evaluated by testing it on a sequence of MRI and $\mathrm{CE}$ images. The algorithm was first tested on single images and then on sequence of images. To obtain the image sequences few medical videos are taken from MR-TIP database and Sundaram Medical Foundation. The image data for testing include few CE image sequences and one set of MRI sequence. The results are evaluated based on Compression Ratio and Peak Signal-to-Noise Ratio. Fig. 4 shows sample MRI Image sequences and Fig.5 shows sample CE Image Sequences. The images in these CE sequences are of dimension 700x278 and the images in these MRI sequences are of dimension 160x194. Motion Estimation and Motion Compensation is applied to these image sequences using Binary Tree Search algorithm and Super-Spatial Structure Compression is applied. The output code of SSP is then applied to LZ8 compression to enhance the compression ratio. The proposed algorithm produced compression ratio on an average of $7.28 \%$ for MRI sequence and $6.55 \%$ for CE sequence. To ensure the quality of the decompressed image PSNR is calculated.
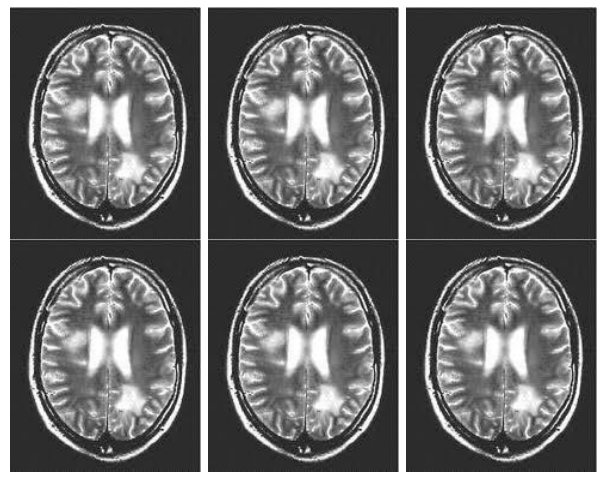

Fig. 4 MRI Image Sequences

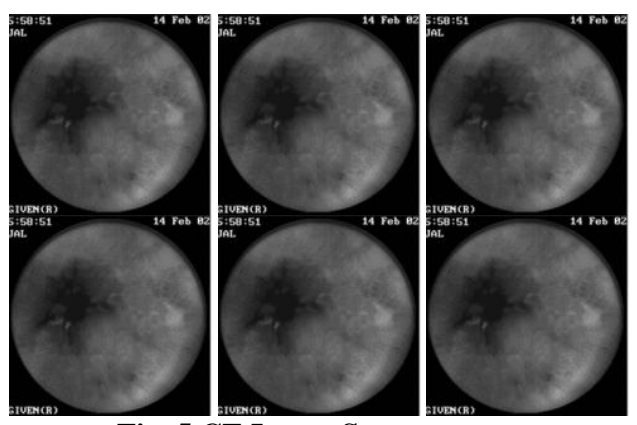

Fig. 5 CE Image Sequences

Table I gives the compression ratio of MRI and CE image sequences. The proposed methodology gives very good result compared to other state-of the art coding. The coding gain provided by the proposed method is significant. The result for each frame is shown in fig 6 and fig 7. From Fig 6 and 7 it is proved that the proposed methodology gives better result than the prior arts.

Table II shows the average PSNR of MRI and CE sequences. The Bits per pixel are varied and the values are obtained. With $0.25 \mathrm{bpp}$ the average PSNR of MRI and CE is $44.32 \mathrm{~dB}$ and $45.97 \mathrm{~dB}$ respectively. With $0.25 \mathrm{bpp}$ the average PSNR of MRI and CE is $47.56 \mathrm{~dB}$ and $48.51 \mathrm{~dB}$ respectively. With 
0.25 bpp the average PSNR of MRI and CE is $50.31 \mathrm{~dB}$ and $51.77 \mathrm{~dB}$ respectively

Table 1. CR of MRI and CE Image Sequences

\begin{tabular}{|c|c|c|c|c|}
\hline \multirow{2}{*}{$\begin{array}{c}\text { Image } \\
\text { Sequence }\end{array}$} & \multicolumn{2}{|c|}{ CR- MRI Sequence } & \multicolumn{2}{c|}{ CR- CE Sequence } \\
\cline { 2 - 5 } Existing & $\begin{array}{c}\text { SSP+BT } \\
\text { S+ LZ8- } \\
\text { Proposed }\end{array}$ & $\begin{array}{c}\text { SSP- } \\
\text { Existing }\end{array}$ & $\begin{array}{c}\text { SSP+BTS+ } \\
\text { LZ8- } \\
\text { Proposed }\end{array}$ \\
\hline F1 & 5.86 & 7.32 & 4.52 & 6.12 \\
\hline F2 & 5.64 & 7.17 & 4.81 & 6.35 \\
\hline F3 & 5.88 & 7.41 & 5.32 & 6.87 \\
\hline F4 & 6.05 & 7.59 & 5.21 & 6.64 \\
\hline F5 & 5.67 & 7.2 & 5.25 & 6.71 \\
\hline F6 & 5.54 & 6.98 & 5.12 & 6.62 \\
\hline AVG & 5.77 & 7.28 & 5.04 & 6.55 \\
\hline
\end{tabular}

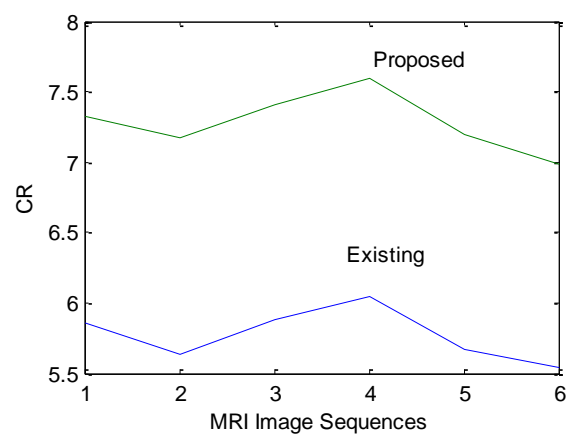

Fig. 6 Compression ratio of MRI image sequences

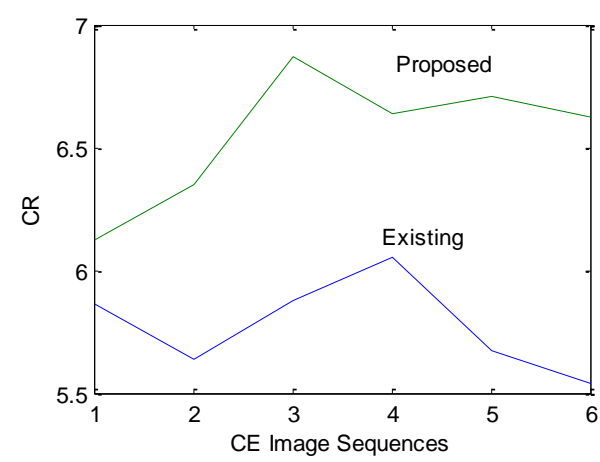

Fig. 7 Compression ratio of $\mathrm{CE}$ image sequences

Table 2. Average PSNR of MRI and CE Image Sequences

\begin{tabular}{|c|c|c|c|c|}
\hline \multirow{2}{*}{$\begin{array}{c}\text { Bits per } \\
\text { pixel } \\
\text { (bpp) }\end{array}$} & \multicolumn{2}{|c|}{ PSNR-MRI Sequence } & \multicolumn{2}{c|}{ PSNR- } \\
\cline { 2 - 5 } Existing & $\begin{array}{c}\text { SSP+BTS+ } \\
\text { LZ8- } \\
\text { Proposed }\end{array}$ & $\begin{array}{c}\text { SSP- } \\
\text { Existing }\end{array}$ & $\begin{array}{c}\text { SSP+BTS+ } \\
\text { LZ8- } \\
\text { Proposed }\end{array}$ \\
\hline 0.25 & 37.18 & 44.32 & 39.89 & 45.97 \\
\hline 0.5 & 40.45 & 47.56 & 42.34 & 48.51 \\
\hline 1 & 43.12 & 50.31 & 45.67 & 51.77 \\
\hline
\end{tabular}

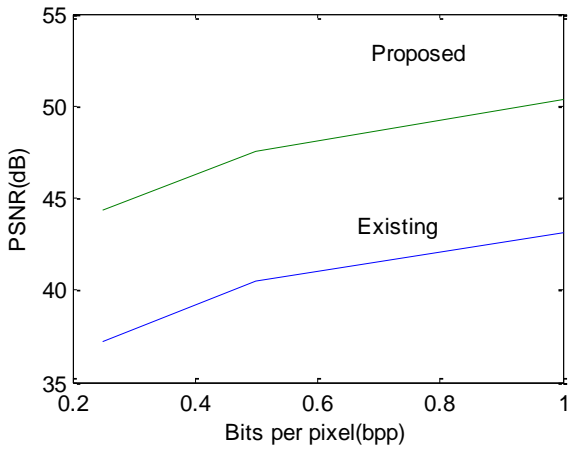

Fig. 8 PSNR of MRI image sequences

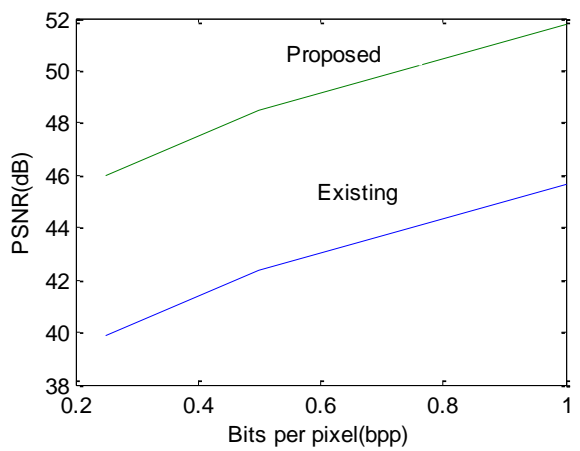

Fig. 9 PSNR of CE image sequences

Fig 8 and 9 shows the PSNR with respect to Bits per pixel. The PSNR of the proposed is higher for both MRI and CE sequences hence the image quality is better compared to other state-of-the-art algorithms.

\section{CONCLUSION}

The algorithm given in this paper makes use of the lossless image compression technique and video compression to achieve higher CR. To achieve high CR the proposed method combines Super-Spatial Structure Prediction (SSP) with interframe coding along with LZ8. The technique used in proposed algorithm gives better result than JPEG-LS and SSP. Fast block-matching algorithm is used here.. In order to have the most accurate searching result and the best compression efficiency we have taken Binary Tree Search (BTS) algorithm for block-matching process. Since this paper exploits interframe correlation in the form of MEMC the proposed is compared with [1][13]. To enhance the compression ratio, SSP is combined with LZ8. From Table I, II and Fig 6,7,8,9 it is analyzed that proposed is much better than other state-ofthe art lossless compression methods.

\section{REFERENCES}

[1] Shaou-Gang Miaou, Fu-Sheng Ke, and Shu-Ching Chen, "A Lossless Compression Method for Medical Image Sequences Using JPEG-LS and Interframe Coding," IEEE Transaction on Information Technology in Biomedicine, vol. 13,No.5,Sep 2009

[2] S.E. Ghare, M.A.Mohd .Ali, K.Jumari and M.Ismail, “An Efficient Low Complexity Lossless Coding Algorithm for Medical Images," in American Journal of Applied Sciences 6 (8): 1502-1508, 2009.

[3] R.Srikanth, A.G.Ramakrishnan, "Context- Interframe Coding of MR Images". 
[4] S.Bhavani, Dr.K.Thanushkodi, "A Survey in Coding Algorithms in Medical Image Compression,"in International Journal on Computer Science and Engineering, vol.02, No.5, 2010, 1429-1434

[5] Xiwen OwenZhao,Zhi haiHenryHe, "Lossless Image Compression Using Super-Spatial Structure Prediction",IEEE Signal Processing,vol.17,no.4,April 2010

[6] C.S.Rawat, Seema G Bhatea, Dr.Sukadev Meher,"A Novel Algoritm of Super-Spatial Structure Prediction for RGB Colourspace",International ournal of Scientific \& Engineering Research, vol.3, Issue 2, February 2012.

[7] M. J. Weinberger, G. Seroussi, and G. Sapiro, "The LOCO-I Lossless Image Compression Algorithm: Principles and standardization into JPEG-LS," IEEE Trans. Image Process., vol.9, no. 8, pp. 1309-1324, Aug. 2000 .

[8] M. Weinberger, G. Seroussi, and G. Sapiro, "LOCO-I: A Low Complexity, Context-based, Lossless Image Compression Algorithm," in Proc. IEEE Data Compression Conf., Snowbird,UT, Mar./Apr. 1996, pp. 140-149.

[9] Y. D. Wang, "The Implementation of Undistorted Dynamic Compression Technique for Biomedical Image," Master's thesis, Dept. Electr. Eng., Nat.Cheng Kung Univ., Taiwan, 2005.
[10] D.Brunello, G.Calvagno, G. A. Mian, and R. Rinaldo, "Lossless Compression of Video using Temporal Information," IEEE Trans. Image Process, vol. 12, no. 2, pp. 132-139, Feb. 2003.

[11] N.D.Memon and Khalid Sayood, "Lossless Compression of Video Sequences," IEEE Trans. Commun., vol.44, no.10, pp.1340-1345.

[12] M. F. Zhang, J. Hu, and L. M. Zhang, "Lossless Video Compression using Combination of Temporal and Spatial Prediction," in Proc. IEEE Int. Conf. Neural Newt. Signal Process. Dec. 2003, vol. 2, pp. 1193-1196.

[13] Mudassar Raza, Ahmed Adnan et.al, "Lossless Compression Method for Medical Image Sequences Using Super-Spatial Structure Prediction and Inter-frame Coding," International Journal of Advanced TResearch and Technology, vol.10,No.4,August 2012.

[14] C. Saravanan and M.Surrendar, "Enhancing Efficiency of Huffman Coding Using Lempel-Ziv Coding for Image Compression," International Journal of Soft Computing and Engineering, ISSN:2231-2307, Vol.2, Issue-6, January 2011

[15] T. Wiegand, G.J.Sullivan et.al., "Overview of the H.264/AVC video coding standard," IEEE Trans.Circuits Systems Video Technology, vol.13,No.7,June 2003.

[16] Juan,Diego et.al., "Pseudo-periodic surrpgate data method on voice signals," 11th International Conference, 2012. 